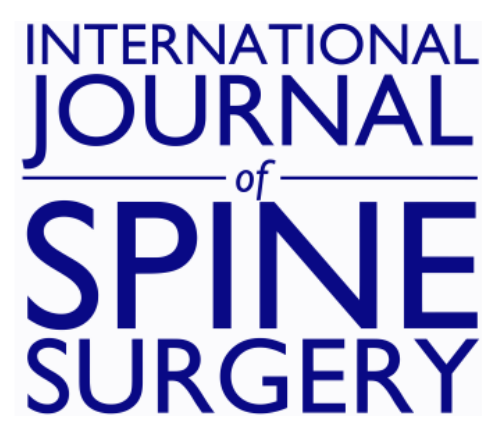

\title{
Benefits of the Paraspinal Muscle-Sparing Approach Versus the Conventional Midline Approach for Posterior Nonfusion Stabilization: Comparative Analysis of Clinical and Functional Outcomes
}

Neel Anand, Eli M. Baron and Robert S. Bray, Jr.

Int J Spine Surg 2007, 1 (3) 93-99

doi: https://doi.org/10.1016/SASJ-2007-0101-RR

http://ijssurgery.com/content/1/3/93

This information is current as of April 26, 2023.

Email Alerts Receive free email-alerts when new articles cite this article. Sign up at:

http://ijssurgery.com/alerts

The International Journal of Spine Surgery

2397 Waterbury Circle, Suite 1,

Aurora, IL 60504, Phone: +1-630-375-1432 


\title{
Benefits of the Paraspinal Muscle-Sparing Approach Versus the Conventional Midline Approach for Posterior Nonfusion Stabilization: Comparative Analysis of Clinical and Functional Outcomes
}

\author{
Neel Anand, MD, Eli M. Baron, MD, Robert S. Bray, Jr., MD
}

\begin{abstract}
Background

The influence of approach on outcomes of posterior nonfusion stabilization has not been described. This paper analyzes the influence of surgical approach on functional outcome with nonfusion stabilization.

Methods

We performed a prospective consecutive cohort outcome analysis of 88 patients who had undergone posterior nonfusion stabilization of the lumbar spine at 178 levels using the Dynesys system (Zimmer Spine, Inc, Warsaw, Indiana). Patients needing decompression $(n=42)$ were operated through a midline approach using microscopic laminotomy/foraminotomy with or without discectomy, followed by posterior nonfusion stabilization with Dynesys. None of the patients had a complete laminectomy. Patients not needing decompression $(n=46)$ underwent the procedure via the bilateral paraspinal muscle-sparing approach and were subsequently stabilized. Clinical and functional outcomes data were collected using the visual analog scale (VAS), Treatment Intensity Score (TIS), Oswestry Disability Index (ODI), and SF-36. Average follow-up was 18 months (range, 12-36 mo).
\end{abstract}

\begin{abstract}
Results
All outcome measures in both groups showed significant improvement at last follow-up. Between the groups a significant difference was apparent in the reduction of the TIS when measured at 1 week and 6 weeks. The preoperative, 1 -week, and 6-week values were 66, 48, and 40, respectively $(P<0.05)$, for the midline group and 80,32 , and $28(P<0.05)$ for the paraspinal group. This trend continued through 3 to 6 months after the procedure but did not reach statistical significance. In the paraspinal group, pain scores showed a nonsignificant trend toward lower values in the first month, compared with values in the midline group. Patients reported excellent to fair results, with the exception of three patients in the midline group and two in the paraspinal group, who rated the procedure as fair.
\end{abstract}

\begin{abstract}
Conclusions
Significantly fewer patients required postoperative narcotics in the paraspinal group than in the midline group. This improvement in early outcomes suggests a significant early benefit to the less tissue-destructive muscle-sparing approach in posterior nonfusion stabilization procedures.
\end{abstract}

Key Words Posterior nonfusion spinal stabilization, muscle-sparing approach, paraspinal approach.SAS Journal. Summer 2007;1:93-99. DOI: SASJ-2007-0101-RR

\section{INTRODUCTION}

Despite satisfactory clinical outcomes having been demonstrated for various minimally invasive spinal procedures, including microendoscopic discectomy ${ }^{1,2}$ and minimally invasive spinal fusion, ${ }^{3-6}$ few existing prospective studies have compared outcomes with the conventional midline approach to those for which a minimally tissue-disruptive approach is taken. ${ }^{7,8}$ Even less is known about the outcomes of posterior nonfusion stabilization patients in which a minimally disruptive approach is taken as opposed to a conventional approach.

Dynesys (Dynamic Neutralization System for the Spine) is a posterior nonfusion stabilization system consisting of pedicle screws, composed of titanium alloy (Protasul100; Zimmer Spine, Warsaw, Indiana) pedicle screws, polyethyleneterephthalate (Sulene-PET, Zimmer Spine) cords, and polycarbonateurethane spacers. ${ }^{9}$ With the Dynesys system, flexion compresses the disc, and the axis of flexion is the posterior ligament. If a patient extends, the anterior annulus opens without compression of the posterior annulus, resulting in unloading of the disc, especially if the patient achieves a position of lordosis, where the spacers become weight bearing. ${ }^{10}$ The system has also been demonstrated to offload the disc at stabilized segments in biomechanical testing. ${ }^{.1}$ Good outcomes have been demonstrated using Dynesys for a variety of degenerative lumbar spine indications. ${ }^{12}$ Screw placement 
without disturbing the facet capsule and joint is a critical part of the posterior nonfusion stabilization system. The screws are placed in a far-out-to-in trajectory, and the paraspinal approach lends itself to the correct trajectory for insertion of appropriate screws and placement of the spacers, which may in itself be an important factor in obtaining clinical outcomes. The Dynesys system is also exacting in its technique for placing of the appropriate spacers, and this, along with the midline approach and inappropriate screw placement, may be the reason for the poor results reported in some series..$^{13}$

We prospectively studied the functional outcome of patients undergoing Dynesys implantation for a variety of degenerative indications. We compared patients undergoing a conventional midline approach with a decompression with patients undergoing a minimally tissue-disruptive paraspinal approach without any decompression. Outcomes were assessed using a variety of questionnaire-based instruments.

\section{METHODS}

Eighty-eight patients underwent posterior nonfusion stabilization of the lumbar spine at 118 levels using the Dynesys system (Zimmer Spine). Study demographics are summarized in Table 1, and indications are outlined in Table 2. The indications were spondylolisthesis in 48 patients, retrolisthesis in 12 patients, central/lateral recess stenosis in 52 patients, dynamic foraminal stenosis in 6 patients, and degenerative disc disease in 36 patients; 4 patients were instrumented adjacent to a previous fusion. All patients were placed prone on a Jackson table with care being taken to maintain lordosis. Patients needing decompression $(n=42)$ were operated through a midline approach with microscopic laminotomy/foraminotomy with or without a discectomy followed by posterior nonfusion stabilization with the Dynesys system. The facet capsule and facet joint were carefully preserved. The midline structures were preserved in all patients, with care being taken to suture the lumbodorsal fascia back to the midline at the end of the procedure. None of the patients had a complete laminectomy. We used a bilateral paraspinal muscle-sparing approach in patients who did not need decompression $(n=46)$. We used anterioposterior fluoroscopy for the paraspinal incisions to mark the lateral edge of each respective pedicle and connect the dots (Figure 1). The intermuscular plane was carefully developed down to the transverse processes of the level being instrumented. We took great care not to violate the facet capsules or any of the muscle attachments. The top of the transverse process near the superior facet lateral edge was exposed. We used a narrowblade self-retaining retractor to maintain the intermuscular plane. The mamillary process was identified and the entrance point marked with a pilot hole to the corresponding pedicle. The pedicle screws were then placed under fluoroscopic control, following which the retractor was moved to the next transverse process and the pedicle was instrumented. Once the screws were in, the retractor was placed between the screws while passing the spacer (Figure 2).
Table 1

\begin{tabular}{lrr}
\hline \multicolumn{3}{c}{$\begin{array}{c}\text { Demographics } \\
88 \mathrm{pts}, 178 \text { levels }\end{array}$} \\
\hline Midline $(n=42)$ & Paraspinal $(n=46)$ \\
Male/Female & $48(32$ to 79$)$ & $43(24$ to 69) \\
W/C & $19 / 23$ & $24 / 22$ \\
Smokers & 0 & 0 \\
Previous surgery & 0 & 0 \\
Note. W/C = Workers' Compensation & 21 & 26 \\
\hline
\end{tabular}

Table 2

\begin{tabular}{lcc}
\hline \multicolumn{3}{c}{$\begin{array}{c}\text { Dynesys Indications } \\
88 \text { pts, 178 levels }\end{array}$} \\
\hline & Midline & Paraspinal \\
\hline & 28 & 2 \\
Spondylolisthesis & 12 & 0 \\
Retrolisthesis & 40 & 0 \\
Lateral/central stenosis & & \\
(Overlapping diagnosis) & 0 & 6 \\
Dynamic foraminal stenosis & 0 & 36 \\
DDD & 2 & 2 \\
Adjacent to fusion & & \\
Note. DDD $=$ degenerative disc disease &
\end{tabular}

\section{Figure 1}

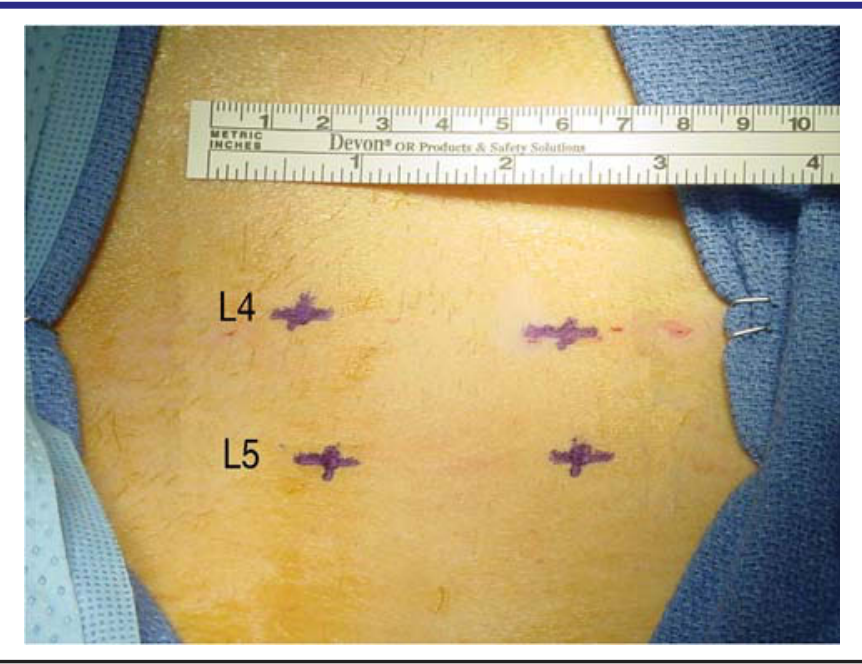

Photograph showing lumbar spine with pedicles marked on the skin per anteroposterior fluoroscopy. A linear mark is then made connecting the lateral edge of the pedicles.

Age, sex distribution, and levels operated were comparable. Smokers and patients receiving Workers' Compensation were excluded. The average follow-up was 18 months, with a minimum 1-year follow-up (12-36 mo).

We collected data prospectively using self-administered questionnaires, including the visual analog scale (VAS), ${ }^{5}$ Treatment Intensity Score (TIS), ${ }^{6,7}$ Oswestry Disability 
Figure 2
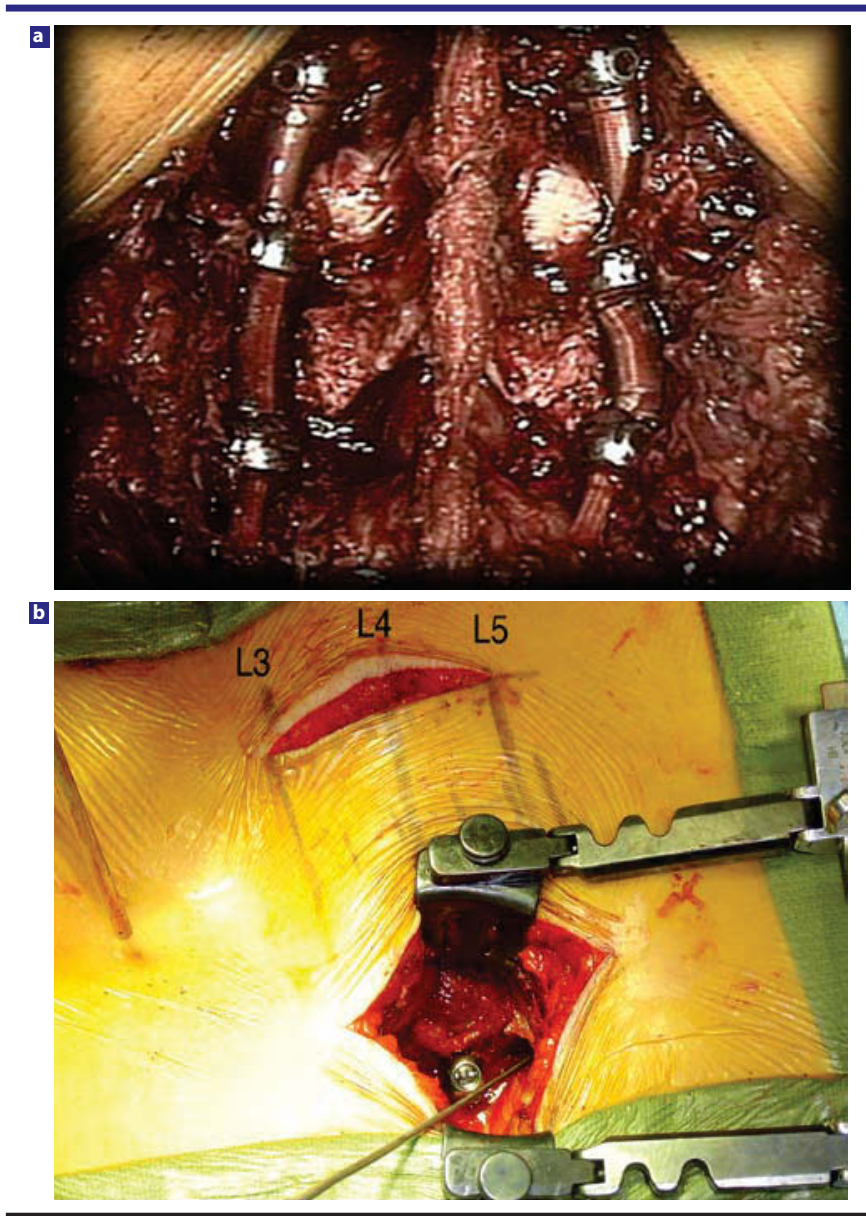

(a) Photograph of Dynesys implanted via the midline approach in cases where a decompression was performed. (b) Photograph of Dynesys implanted via musclesparing approach.

Index (ODI), ${ }^{8}$ and SF-36. ${ }^{9}$ The patients completed the questionnaires before the operation and at every subsequent visit postoperatively. Follow-up was at 1 week, 6 weeks, 3 months, 6 months, 12 months, 18 months, and 2 years. We followed the patients regularly for 2 years and once a year thereafter. Patient satisfaction and recommendations were also documented.

\section{Statistical Methods}

All data were entered into a database and collected on an ongoing basis. The Student's t test (of the two-sample unequal variance) was performed for statistical analysis with significance set at $P \leq 0.05$.

\section{RESULTS}

There were no major vascular or neurological complications. The surgical time was 150 minutes, with estimated blood loss of $350 \mathrm{~mL}$ for the midline approach; for the paraspinal approach, surgical time and blood loss were 120 minutes and $300 \mathrm{~mL}$, respectively (Table 3).

All outcome measures in both groups showed significant improvement at last follow-up. We observed a significant difference
Table 3

\begin{tabular}{lcc}
\hline & $\begin{array}{c}\text { Surgical Details } \\
(88 \text { pts, } 178 \text { levels })\end{array}$ \\
\hline Operative time & $150(120-240)$ & $120(90-180)$ \\
Hospital stay & 2.6 days $(1$ to 5 days $)$ & 1.8 days $(1$ to 3 days $)$ \\
Blood loss & $350(100-500)$ & $300(50-400)$ \\
& Levels stabilized & 16 \\
One & 15 & 26 \\
Two & 23 & 4 \\
Three & 4 & \\
Follow-up & 18 months $(12$ months to 36 months $)$ \\
\hline
\end{tabular}

between the groups in the reduction of TIS when measured at 1 week and 6 weeks. The preoperative 1-week and 6-week values for the midline group were 66,48 , and 40 ; values for the paraspinal group were 80,32 , and 28 ( $P<0.05$ for both groups) (Figure 3 ). This trend continued postoperatively for 3 to 6 months but was not statically significant. At 12 and 24 months no difference was evident between the two groups. VAS scores in the paraspinal group showed a nonsignificant trend to lower values during the first month compared with VAS scores in the midline group (Table 4).

\section{Figure 3}

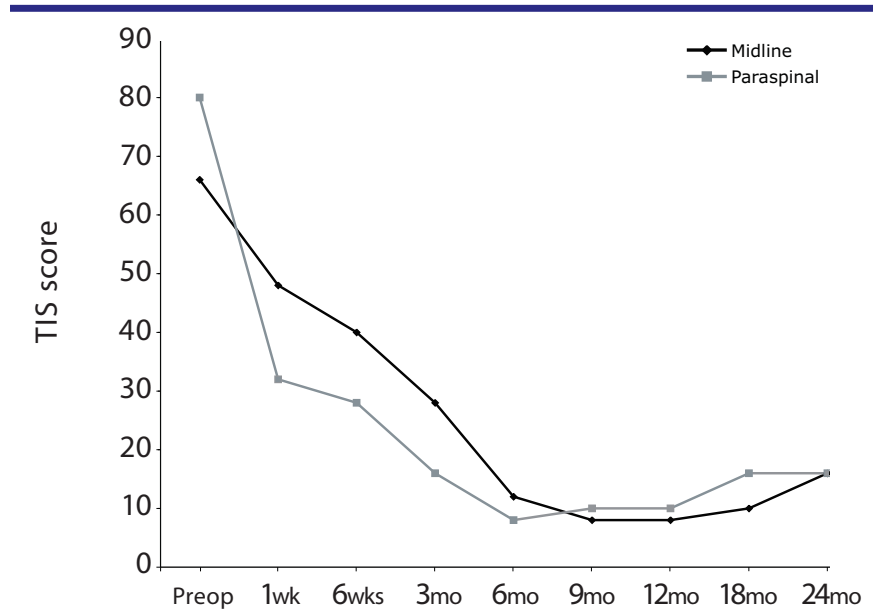

Time after surgery

$P<0.05$ at 1 to 6 weeks postop, TIS $=$ Treatment Intensity Score

Graph showing TIS significantly improved at 6 weeks for patients who underwent posterior nonfusion stabilization via paraspinal muscle-sparing approach versus the midline muscle-stripping approach.

The ODI and SF-36 scores identified no significant difference between the groups (Table 4). Three patients in the midline group rated the procedure as fair, as did two in the paraspinal group. The rest rated it excellent or good, and none rated it poor. Twenty-nine of 31 patients in the midline group were satisfied and would recommend this approach, compared with 34 of 36 in the paraspinal group. 
Table 4

VAS, ODI and SF-36 Physical Component Scores in Patients Undergoing Posterior Nonfusion Stabilization

\begin{tabular}{|c|c|c|c|c|c|c|c|c|c|}
\hline & \multicolumn{3}{|c|}{ VAS Scores } & \multicolumn{3}{|c|}{ ODI } & \multicolumn{3}{|c|}{ SF-36 Physical Component Score } \\
\hline & Midline & Paraspinal & $P$ value & Midline & Paraspinal & $P$ value & Midline & Paraspinal & $P$ value \\
\hline Pre-op & 65 & 90 & & 55 & 68 & & 34 & 28.56 & \\
\hline 6 weeks & 35.62 & 30 & 0.64 & 50 & 50 & 0.50 & 30.38 & 30.38 & 0.50 \\
\hline 3 months & 30.63 & 20 & 0.82 & 29.1 & 25 & 0.62 & 36.78 & 36.78 & 0.42 \\
\hline 6 months & 30.81 & 25 & 0.93 & 25.6 & 25 & 0.62 & 36 & 36 & 0.39 \\
\hline 12 months & 24 & 20 & 0.98 & 20.2 & 20 & 0.61 & 38 & 35.4 & 0.21 \\
\hline 18 months & 22 & 25 & 0.95 & 22 & 22 & 0.60 & 38 & 34 & 0.09 \\
\hline 2 years & 24 & 22 & 1.00 & 20.4 & 22 & 0.52 & 36 & 34 & 0.05 \\
\hline
\end{tabular}

Three patients were reoperated, all in the paraspinal group-one for a misplaced screw and the other for extension to the next level. One patient had a conversion to a fusion due to recurrence of discogenic back pain after 3 months of symptomatic relief. All studies, including radiographs, CT scans, and MRIs, showed no loose or misplaced screws. At exploration 9 months after the surgery, the locking nut to one of the screws was found to be loose and the spacer was slack and had minimal tension. Because the decision to fuse was made before the original surgery, the level was fused postoperatively with a cantilever transforaminal lumbar interbody fusion (TLIF) procedure. ${ }^{6}$

Three patients in the midline group needed postoperative selective nerve root blocks, whereas two in the paraspinal group needed facet blocks. No patient has shown evidence of screw loosening or screw failure on plain films and dynamic films at last follow-up. A CT scan was ordered for any instance in which the status of a screw was questionable. Fifteen patients had CT results that demonstrated no loosening.

\section{DISCUSSION}

Wiltse et al. ${ }^{1,10}$ popularized the paraspinal sacrospinalissplitting approach to the lumbar spine. In his original report on this procedure, Wiltse ${ }^{1}$ described the approach as passing "trans-sacrospinalis. The sacrospinalis is split about two-finger breadths lateral to the midline. He noted further: "the muscle fibers do not split cleanly since at this level they run in various directions." Wiltse argued that this approach offered a more direct route to the transverse processes and facets of the lumbar spine, with less bleeding.

Nevertheless, the exact location of the sacrospinalis muscle requiring splitting remains unclear despite Wiltse's description. Vialle et al. ${ }^{11}$ proposed, on the basis of cadaver studies, that the splitting should be done at the natural cleavage plane between the multifidus and the longissimus parts of the sacrospinalis. We prefer the term "modified muscle-sparing approach," in which a Langenback elevator is used to tease the fibers of the multifidus medially so the cleavage plane can be clearly

\section{Figure 4}

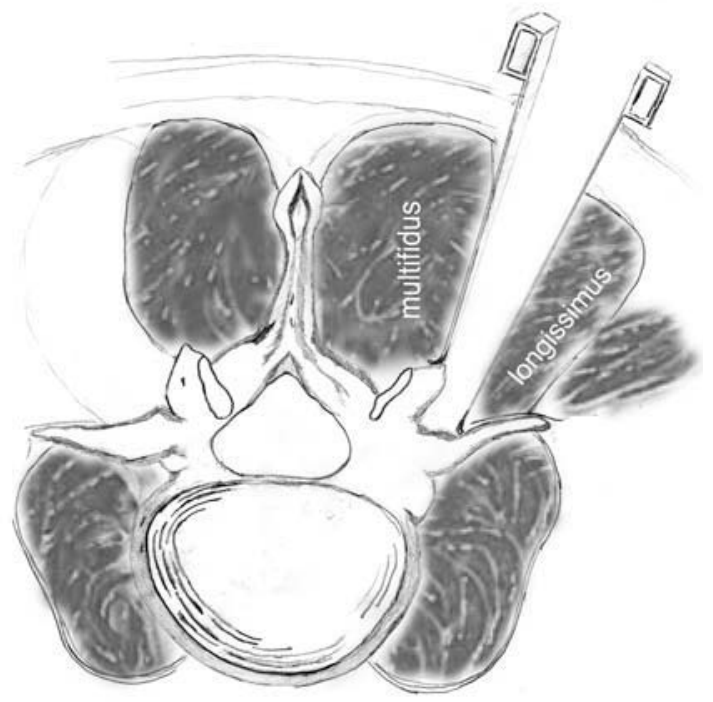

Illustration showing the modified Wiltse muscle-sparing approach. Note the approach between the multifidus and longissimus muscles.

visualized (Figure 4). Subsequently, we use a narrow-blade McCulloch retractor with a deep blade laterally and a shorter blade medially for retraction directly over the transverse process. In our experience this procedure can be done quite cleanly. We have encountered minimal to no muscle bleeding using this approach. Others use the term "muscle sparing" in reference to a paraspinal approach using a tubular retractor. ${ }^{3}$ We prefer to use this term for a modified Wiltse approach where muscle fibers are not grossly disrupted.

The benefits of this minimally disruptive approach might include decreased damage to the paraspinal musculature, which in turn could lead to improved outcomes. Several authors have described paraspinal muscle damage seen after midline, muscle-stripping approaches. ${ }^{12-18}$ Higher levels of inflammatory mediators have been demonstrated in patients undergoing conventional microdiscectomy as opposed to minimally invasive microdiscectomy. ${ }^{19}$ Similarly, MRI has 
revealed increased edema in the paraspinal muscles of patients in whom the midline muscle-stripping approach was used as opposed to a minimally invasive approach (Figure 5). ${ }^{20}$

\section{Figure 5}

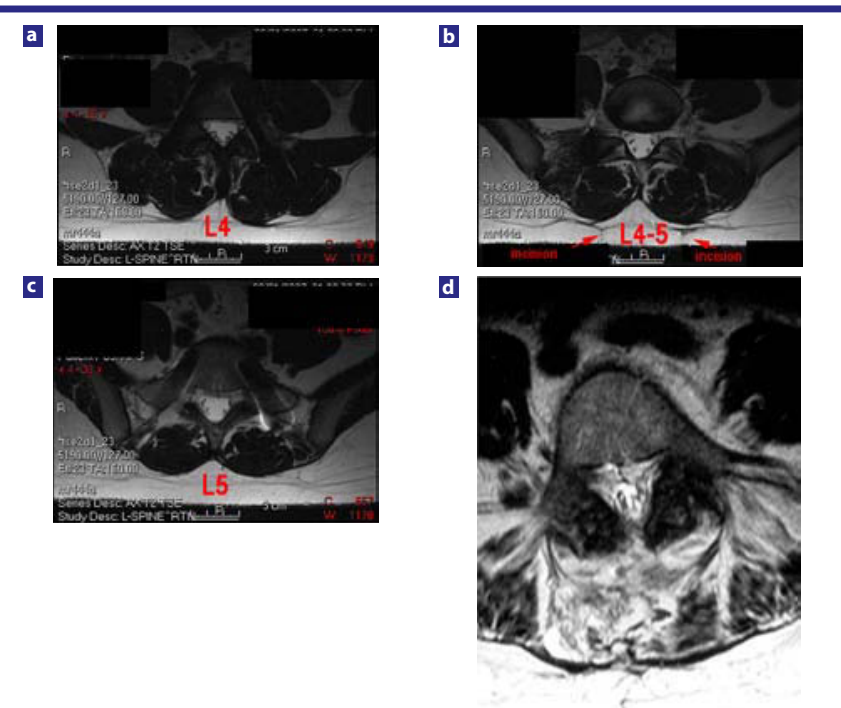

(a), (b), (c). T2 weighted MRI of patients showing changes in the paraspinal muscles of a patient undergoing posterior nonfusion stabilization via the anatomy-preserving muscle-sparing approach vs (d), typical changes seen in a midline muscle stripping approach. Note the extensive high signal seen in the paraspinal muscles of the patient undergoing the midline approach.

Panjabi ${ }^{21}$ discussed the concept of the neutral zone in the lumbar spine as a region of intervertebral motion around the neutral posture, where little resistance is offered by the passive spinal column. ${ }^{21}$ Panjabi also observed that the neutral zone may be a clinically important measure of spinal stability function. The area of the neutral zone may increase with injury or degeneration of the spinal column or with weakness of the muscles, which in turn may lead to spinal instability or a low-back problem. Panjabi thought an increase in the neutral zone in turn increased the stress on the surrounding musculature to maintain the neutral zone. This increased muscle stress could be a reason for back pain. Panjabi concluded that the spinal stabilizing system adjusts so that the neutral zone remains within certain defined physiologic thresholds to avoid clinical instability. Hence, in nonfusion surgery, maintaining the soft tissue and musculature surrounding the spinal segment may be far more relevant clinically than in fusion surgery to achieve segmental balance and maintain the neutral zone within physiologic limits. This approach may, in turn, improve outcomes.

Despite the satisfactory clinical outcomes demonstrated for various minimally invasive spinal procedures, including microendoscopic discectomy ${ }^{22,23}$ and minimally invasive spinal fusion, ${ }^{2,3,24,25}$ few prospective studies have compared outcomes using the conventional midline approach to results of a minimally tissue-disruptive approach. ${ }^{26,27}$ We know even less about the outcomes of posterior nonfusion stabilization in a minimally disruptive approach as opposed to a conventional approach.
Dynesys is a posterior nonfusion stabilization system composed of titanium alloy (Protasul-100) pedicle screws, polyethyleneterephthalate (Sulene-PET) cords, and polycarbonateurethane spacers. ${ }^{28}$ With the Dynesys system, flexion compresses the disc, and the axis of flexion is the posterior ligament. If a patient extends, the anterior annulus opens without compression of the posterior annulus, resulting in unloading of the disc, especially if the patient achieves a position of lordosis, in which the spacers become weight bearing. ${ }^{29}$ In biomechanical testing, the system demonstrated its capability to offload the disc at stabilized segments. ${ }^{30}$ Dynesys has led to good outcomes in a variety of degenerative lumbar spine indications. ${ }^{31}$ Screw placement without disturbing the facet capsule and joint is a critical part of the posterior nonfusion stabilization system. The screws are placed in a far-out-to-in trajectory, and the paraspinal approach lends itself to the correct trajectory for insertion of appropriate screws and placement of the spacers. This feature may be important for obtaining clinical outcomes. The Dynesys system is also exacting in its technique for placement of the appropriate spacers; along with the midline approach and inappropriate screw placement, this may be the reason for the poor results reported in some series. ${ }^{32}$

We prospectively studied the functional outcome of patients undergoing Dynesys implantation for the degenerative indications described previously (see METHODS). We compared patients undergoing a conventional midline approach with decompression to patients undergoing a minimally tissuedisruptive paraspinal approach without decompression. We assessed the outcomes using a variety of questionnaire-based instruments.

For the comparison we used the TIS, a self-administered questionnaire instrument, to assess patient use of analgesic agents, medical treatments, and alternative medical treatment for pain. ${ }^{6,7}$ Patients undergoing the modified Wiltse musclesparing approach had significantly reduced requirements for pain interventions and analgesics at 1 week and 6 weeks postoperatively. These findings are consistent with those of Schwender et al., ${ }^{3}$ who reported very short stays and marked reduction in narcotic use in patients undergoing minimally invasive TLIF. Although the trend for improved TIS scores continued at 3 to 6 months after surgery, it was no longer statistically significant. At 1 year both groups had nearly identical TIS scores.

VAS scores improved more in the muscle-sparing group but not to a significant extent (Table 4). This may be because both groups had reduced pain but the midline approach patients used more medication to achieve the same degree of pain reduction. The TIS was able to capture this difference objectively. ODI and SF-36 scores improved in both groups, with no significant differences between the groups. The ODI seems more reliable in capturing long-term disability, ${ }^{33}$ and as expected, did not capture any differences in the short term. We were unable 
to show any differences in long-term disability between the groups. A larger cohort with longer follow-up will be needed to sort out any differences. The SF-36 is an overall assessment of functional health and well-being for which a total score is divided into a physical and mental health score. Each score is divided into four subcomponents measuring such items as general health and social functioning. ${ }^{34}$ Nevertheless, the SF36 may underestimate changes in quality of life associated with spine surgery, as three of its eight scales have high floor/ceiling effects. Thus, summary scores from the SF-36 assessment may not identify significant differences between groups undergoing surgical interventions. ${ }^{35}$

We are not certain how to explain the fact that all three reoperations were in the paraspinal group. Any of them could very well have been in the midline group. We believe the occurrence of the reoperations in the paraspinal group is coincidental. A larger cohort should be able to sort out these differences.

Weaknesses in this study include its limited number of patients; the fact that patients in the midline group also underwent decompression; and the heterogeneous, nonrandomized patient population. Grob et al. ${ }^{32}$ noted that patients who underwent Dynesys and decompression did better than those with only Dynesys instrumentation. ${ }^{32}$ All patients were operated through a midline approach with dissection of the erector spinae muscles. Our series showed a significant reduction in narcotic requirements when Dynesys instrumentation was placed through a paraspinal muscle-sparing approach. The fact that all our patients who did not need a decompression had a paraspinal approach may indicate that the paraspinal approach is better for posterior nonfusion stabilization, especially when decompression is not carried out. The significantly better results compared with outcomes for instrumentation and decompression through the midline approach (in contrast to results in the Grob et al. study) suggest that the paraspinal approach warrants further investigation for its potential as the treatment of choice with pedicle-based posterior nonfusion stabilization. The tissuesparing effects may be more profound with longer-term (5 years or more) follow-up because maintaining the neutral zone within physiologic limits and soft tissue segmental balance may be important factors in posterior nonfusion stabilization.

We acknowledge that the differences in the groups studied, whereby one group underwent decompression and the other did not, are limitations. Nevertheless, despite decompression and relief of leg pain in the midline group, patients needed increased amounts of narcotics to control their back pain, as compared with the paraspinal group. This we feel supports the paraspinal approach for posterior nonfusion stabilization. We also hypothesize that the tissue-sparing effects may be more profound with longer-term (5 years or more) follow-up, as maintaining the neutral zone within physiological limits and soft tissue segmental balance may be important factors in posterior nonfusion stabilization.
Posterior nonfusion stabilization has been performed for a variety of lumbar degenerative conditions that have been traditionally treated with lumbar fusion. A muscle-sparing modified Wiltse paraspinal approach may be associated with improved 6-week outcomes and reduced use of narcotics over the traditional muscle-stripping midline approach.

Neel Anand, MD, Eli M. Baron, MD, Robert S. Bray, Jr., MD From the CedarsSinai Institute for Spinal Disorders, Los Angeles, CA

Address correspondence and reprint requests to Neel Anand, MD, Cedars-Sinai Institute for Spinal Disorders, 444 S. San Vicente Boulevard, Suite 800, Los Angeles, CA 90048. (email: Neel.Anand@cshs.org)

This submission was received June 15, 2007 and accepted for publication August 24, 2007.

The authors' study received IRB approval.

Dr. Anand participates in the Speakers' Bureau for Zimmer Spine.

\section{REFERENCES}

1. Wiltse LL. The paraspinal sacrospinalis-splitting approach to the lumbar spine. Clin Orthop Relat Res. 1973;91:48-57.

2. Tuttle J, Shakir A, Choudhri HF. Paramedian approach for transforaminal lumbar interbody fusion with unilateral pedicle screw fixation. Technical note and preliminary report on 47 cases. Neurosurg Focus (Electronic). 2006;20:E5.

3. Schwender JD, Holly LT, Rouben DP, Foley KT. Minimally invasive transforaminal lumbar interbody fusion (TLIF): technical feasibility and initial results. J Spinal Disord Tech. 2005;18(Suppl):S1-S6.

4. Holly LT, Schwender JD, Rouben DP, Foley KT. Minimally invasive transforaminal lumbar interbody fusion: indications, technique, and complications. Neurosurg Focus (Electronic). 2006;20:E6.

5. Chapman CR, Casey KL, Dubner R, Foley KM, Gracely RH, Reading AE. Pain measurement: an overview. Pain. 1985;22:1-31.

6. Anand N, Hamilton JF, Perri B, Miraliakbar H, Goldstein T. Cantilever TLIF with structural allograft and RhBMP2 for correction and maintenance of segmental sagittal lordosis: long-term clinical, radiographic, and functional outcome. Spine. 2006;31:E748-753.

7. Anand N, Regan JJ, Bray RS. Cervical spine disability questionnaire and treatment intensity question: Objective outcome measures of cervical spine functionial status. An analysis of psychometric properties. Presented at meeting of the Cervical Spine Research Society, December 1-2 2001, in Monterey, California.

8. Fairbank JC, Couper J, Davies JB, O'Brien JP. The Oswestry low back pain disability questionnaire. Physiotherapy. 1980;66:271-273.

9. Ware JE, Jr., Sherbourne CD. The MOS 36-item short-form health survey (SF-36). I. Conceptual framework and item selection. Med Care. 1992;30(6):473-483.

10. Wiltse LL, Bateman JG, Hutchinson RH, Nelson WE. The paraspinal sacrospinalis-splitting approach to the lumbar spine. J Bone Joint Surg Am. 1968;50:919-926. 
11. Vialle R, Wicart P, Drain O, Dubousset J, Court C. The Wiltse paraspinal approach to the lumbar spine revisited: an anatomic study. Clin Orthop Relat Res. 2006;445:175-180.

12. Suwa H, Hanakita J, Ohshita N, Gotoh K, Matsuoka N, Morizane A. Postoperative changes in paraspinal muscle thickness after various lumbar back surgery procedures. Neurol Med Chir (Tokyo). 2000;40:151-154; discussion 154-155.

13. Gejo R, Matsui H, Kawaguchi Y, Ishihara H, Tsuji H. Serial changes in trunk muscle performance after posterior lumbar surgery. Spine. 1999;24:1023-1028.

14. Kawaguchi Y, Yabuki S, Styf J, et al. Back muscle injury after posterior lumbar spine surgery. Topographic evaluation of intramuscular pressure and blood flow in the porcine back muscle during surgery. Spine. 1996;21:2683-2688.

15. Kawaguchi Y, Matsui H, Tsuji H. Back muscle injury after posterior lumbar spine surgery. Part 2: Histologic and histochemical analyses in humans. Spine. 1994;19:2598-2602.

16. Mayer TG, Vanharanta H, Gatchel RJ, et al. Comparison of CT scan muscle measurements and isokinetic trunk strength in postoperative patients. Spine. 1989;14:33-36.

17. Rantanen J, Hurme M, Falck B, et al. The lumbar multifidus muscle five years after surgery for a lumbar intervertebral disc herniation. Spine. 1993;18(5):568-574.

18. Sihvonen T, Herno A, Paljarvi L, et al. Local denervation atrophy of paraspinal muscles in postoperative failed back syndrome. Spine. 1993;18(5):575-581.

19. Sasaoka R, Nakamura H, Konishi S, et al. Objective assessment of reduced invasiveness in MED. Compared with conventional one-level laminotomy. Eur Spine J. 2006;15:577-582.

20. Stevens KJ, Spenciner DB, Griffiths KL, et al. Comparison of minimally invasive and conventional open posterolateral lumbar fusion using magnetic resonance imaging and retraction pressure studies. J Spinal Disord Tech. 2006;19:77-86.

21. Panjabi MM. The stabilizing system of the spine. Part II. Neutral zone and instability hypothesis. J Spinal Dis. 1992;5:390-396; discussion 397.

22. Palmer S. Use of a tubular retractor system in microscopic lumbar discectomy: 1 year prospective results in 135 patients. Neurosurg Focus. 2002;13:E5.

23. Le H, Sandhu FA, Fessler RG. Clinical outcomes after minimal-access surgery for recurrent lumbar disc herniation. Neurosurg Focus. 2003;15: E12.

24. Deutsch H, Musacchio MJ, Jr. Minimally invasive transforaminal lumbar interbody fusion with unilateral pedicle screw fixation. Neurosurg Focus. 2006;20:E10.

25. Jang JS, Lee SH. Minimally invasive transforaminal lumbar interbody fusion with ipsilateral pedicle screw and contralateral facet screw fixation. J Neurosurg Spine. 2005; 3:218-223.

26. Gerszten PC, Welch WC. Spine: minimally invasive techniques. Prog Neurol Surg. 2006;19:135-151.

27. Lehman RA, Jr., Vaccaro AR, Bertagnoli R, Kuklo TR. Standard and minimally invasive approaches to the spine. Orthop Clin North Am. 2005;36:281-292.
28. Schwarzenbach O, Berlemann U, Stoll TM, Dubois G. Posterior dynamic stabilization systems: DYNESYS. Orthop Clin North Am. 2005;36:363-372.

29. Mulholland RC, Sengupta DK. Rationale, principles and experimental evaluation of the concept of soft stabilization. Eur Spine $J$. 2002;11(Suppl 2):S198-S205.

30. Schmoelz W, Huber JF, Nydegger T, Claes L, Wilke HJ. Influence of a dynamic stabilisation system on load bearing of a bridged disc: an in vitro study of intradiscal pressure. Eur Spine J. 2006;15:1276-1285.

31. Stoll TM, Dubois G, Schwarzenbach O. The dynamic neutralization system for the spine: a multi-center study of a novel non-fusion system. Eur Spine J. 2002;11 (Suppl 2):S170-S178.

32. Grob D, Benini A, Junge A, Mannion AF. Clinical experience with the Dynesys semirigid fixation system for the lumbar spine: surgical and patient-oriented outcome in 50 cases after an average of 2 years. Spine. Feb 1 2005;30:324-331.

33. Fairbank JC, Pynsent PB. The Oswestry Disability Index. Spine. 2000;25:940-2952; discussion 2952.

34. Ware JE, Jr. SF-36® Health Survey Update; Lincoln, RI: Quality Metric; 2002

35. Baron R, Elashaal A, Germon T, Hobart J. Measuring outcomes in cervical spine surgery: think twice before using the SF-36. Spine. 2006;31:2575-2584. 